\section{Impacto de la reforma en la legislación sobre consumo de alcohol y conducción en Guadalajara y Zapopan, Jalisco, México: una mirada en el corto plazo}

\author{
Short-term impact of changes in drinking-and- \\ driving legislation in Guadalajara and Zapopan, \\ Jalisco, Mexico \\ Impacto da reforma da legislação sobre consumo \\ de álcool e direção em Guadalajara e Zapopan, \\ Jalisco, México: um olhar de curto prazo
}

\begin{abstract}
The municipalities of Guadalajara and Zapopan, Jalisco State, Mexico, have participated in efforts to reduce road traffic injuries. They have participated actively in the Mexican Road Safety Initiative since 2008. As a result, in September 2010 they passed laws to reduce the legal alcohol levels for driving motor vehicles. To assess the short-term impact of these measures on rates and severity of alcohol-related collisions and injuries, we conducted a secondary analysis of official databases on mortality, morbidity, and collisions. We performed a time-series analysis to assess the trend. Significant changes were observed in the monthly proportion of alcoholrelated deaths and collision rates following these interventions. The article concludes with recommendations to improve the reform's enforcement and results.
\end{abstract}

Alcohol Drinking; Traffic Accidents; Public Policies
Lourdes Gómez-García 1

Ricardo Pérez-Núñez 1

Elisa Hidalgo-Solórzano ${ }^{1}$

\section{Resumen}

Los municipios de Guadalajara y Zapopan, Jalisco, México, han participado en los esfuerzos para reducir la incidencia de las lesiones causadas por el tránsito a lo largo de los años. Así, han sido partícipes de la Iniciativa Mexicana de Seguridad Vial desde 2008, y en septiembre de 2010 entró en vigor la reducción del límite legal de alcoholemia para conducir vehículos de motor. Con el fin de evaluar el impacto en el corto plazo de estas dos acciones en la ocurrencia de colisiones y lesiones, relacionadas con el consumo de alcohol, así como la gravedad de las mismas, se realizó un análisis secundario de las bases de datos oficiales de mortalidad, morbilidad y eventos viales. Se realizó un análisis de series de tiempo, con el objetivo de analizar la tendencia. Se observaron cambios significativos en el porcentaje mensual de muertes asociadas con alcohol y en la tasa de choques a partir de la aplicación de estas intervenciones. Se realizan diversas recomendaciones para mejorar la aplicación y resultados de esta reforma.

Consumo de Bebidas Alcohólicas; Accidentes de Tránsito; Políticas Públicas 


\section{Introducción}

De acuerdo con la Organización Mundial de la Salud (OMS), durante 2010 se registraron 1,24 millones de muertes asociadas al tránsito en el mundo; $80 \%$ de ellas sucedieron en países de ingresos bajos y medios. La tasa de mortalidad global se estimó en 18 por cada 100.000 habitantes 1 . En México, las lesiones causadas por el tránsito fueron la sexta causa de muerte en 2011, con una tasa de mortalidad de 14 fallecidos por cada 100.000 habitantes 2 ; aunque esta tasa pudiera estar subestimada hasta en un $30 \%$ por problemas en la codificación de las muertes en el país 3 .

Las lesiones causadas por el tránsito son un fenómeno multicausal, donde se combinan diversos factores de riesgo. Uno de ellos es el consumo de alcohol, que además influye en la severidad de las lesiones resultantes 1 . Mediante estudios experimentales y epidemiológicos, se ha documentado que existe una relación dosisrespuesta entre la cantidad de alcohol consumido y la ocurrencia de un evento de tránsito ${ }^{4}$. El alcohol afecta las capacidades psicomotoras, la respuesta a los estímulos es más lenta, disminuye la coordinación y la percepción visual. Influye en el comportamiento de los individuos, pues genera una sensación subjetiva de seguridad, lo cual los motiva a tomar conductas de riesgo 5 .

Debido a que el alcohol produce efectos diferenciales en los sujetos, determinar qué nivel de alcohol en sangre es el que afecta a la mayoría de los conductores es una dificultad a la que se han enfrentado los tomadores de decisiones. La evidencia generada muestra que dicho nivel es de $0,05 \mathrm{~g} / \mathrm{dL}$. Los análisis realizados, en torno a la decisión de establecerlo como un límite legal, muestran que es una estrategia adecuada para reducir la frecuencia de conducción después de consumir alcohol ${ }^{6}$, tiende a ser socialmente aceptado y ser costo-efectiva ${ }^{7}$. Al reducir los límites legales de alcoholemia para los conductores de vehículos de motor, la frecuencia de las colisiones, lesiones y muertes asociadas con el consumo de alcohol, se reduce 8 . Tales medidas, incluso desalientan el conducir alcoholizado y cambian los hábitos de consumo de alcohol en los conductores 9 . Todo esto ha motivado a la OMS a promover la implementación de dichas legislaciones y, particularmente, su correcta aplicación 10 . A pesar de ello, pocos países de América han logrado tener legislaciones integrales 11.

En México, la legislación sobre consumo de alcohol y conducción se establece a nivel local; en la mayoría de las entidades federativas el nivel permitido es de $0,08 \mathrm{~g} / \mathrm{dL}^{1}$. Se ha documentado que alrededor de $20 \%$ de los conductores a quienes se les realizó una alcoholimetría dieron posi- tivo; un 3\% estuvieron por encima de los límites permitidos 12 . En los eventos de tránsito; un $10 \%$ de los conductores responsables de choques registrados en vías urbanas y suburbanas de 1999 a 2011 en México fueron reportados con aliento alcohólico (Instituto Nacional de Estadística y Geografía. Accidentes de Tránsito Terrestre en Zonas Urbanas y Suburbanas, 1999-2010. http:// www.inegi.org. $\mathrm{mx} /$ sistemas/olap/proyectos/ bd $/$ consulta.asp? $\mathrm{p}=14744 \& \mathrm{c}=23705 \& \mathrm{~s}=\mathrm{est} \& \mathrm{cl}$, accedido el Abr/2013). Por su parte, información del Sistema de Vigilancia Epidemiológica de las Adicciones (SISVEA) permite observar que un 9\% de los lesionados que utilizaron servicios de urgencias y un $20 \%$ de las personas que fallecieron en México por esta causa de 1994 a 2010 habían consumido alcohol 13 .

En respuesta a esta problemática, durante marzo 2008 se puso en marcha la Iniciativa Mexicana de Seguridad Vial (IMESEVI) en distintas ciudades del país. Esta iniciativa multisectorial tiene como objetivo disminuir las lesiones y muertes relacionadas con el tránsito 14. IMESEVI consta de diversas estrategias, entre ellas, la comunicación social, la promoción de modificaciones a los marcos legales vigentes y la participación ciudadana 12 . Uno de los factores de riesgo a los que se ha dado prioridad es la conducción bajo los efectos del alcohol.

Los municipios de Guadalajara y Zapopan, Jalisco, participaron en la IMESEVI desde su inicio y han logrado dar continuidad a este esfuerzo a lo largo de los años. Como parte del compromiso y liderazgo de las autoridades locales para dar respuesta a las recomendaciones nacionales e internacionales, se propusieron una serie de reformas a la Ley de los Servicios de Vialidad, Tránsito y Transporte del Estado de Jalisco que entraron en vigor a partir de septiembre de 2010. La nueva ley, denominada "Ley Salvavidas", reduce el límite máximo de alcoholemia permitido para conducir vehículos de motor. Previo a la reforma, si al conductor se le detectaba una concentración de alcohol de $0,1 \mathrm{~g} / \mathrm{dL}$, se le multaba con 30 días de salario mínimo, (equivalente a 132,57 dólares aproximadamente) (Organisation for Economic Co-operation and Development. OECD Stat Extracts. PPPs and Exchange Rates. http://stats. oecd.org/Index.aspx?DataSetCode=SNA_TA BLE4, accedido el Abr/2013). A partir de septiembre de 2010, si al conductor se le detecta una alcoholemia entre 0,05 y $0,08 \mathrm{~g} / \mathrm{dL}$, se le atribuye una multa de 150 a 200 días de salario mínimo, (equivalente a 662,87 y hasta 883,82 dólares). Si la alcoholemia detectada se encuentra entre 0,081 y $0,13 \mathrm{~g} / \mathrm{dL}$; además de la sanción económica se retira el vehículo; y si la alcoholemia es superior a $0,13 \mathrm{~g} / \mathrm{dL}$, el conductor y el vehículo son puestos 
a disposición de las autoridades. Como estrategia para reforzar la aplicación de esta legislación, se han puesto en marcha puntos de chequeo de alcoholimetría, principalmente, durante los fines de semana.

Ante el problema de salud pública que representan las lesiones causadas por el tránsito, particularmente aquellas que se relacionan con el consumo de alcohol, el implementar acciones que se conoce son efectivas es parte de la solución. Sin embargo, esta evidencia proviene de países de ingresos altos, con pocas pruebas sobre su efecto en países de ingresos medios y bajos. Esta información idealmente deberá ser sensible al contexto y a las características específicas del país. Asimismo, es necesario generar información que permita conocer si la reducción en el límite legal de alcoholemia tiene repercusiones positivas en la salud y en las condiciones de vida de la población objetivo. Ante la reducción de los límites de alcoholemia en el estado de Jalisco, nos centramos en evaluar su impacto, un año y medio después de entrar en vigor, en términos de reducción en el número de eventos viales, lesionados y fallecidos en los municipios de Guadalajara y Zapopan, pertenecientes al área metropolitana de Guadalajara.

\section{Material y métodos}

\section{Diseño y población de estudio}

Para evaluar el efecto de la "Ley Salvavidas", se realizó un análisis de series de tiempo, empleando información secundaria de las bases de datos oficiales de mortalidad, egresos hospitalarios, colisiones de tránsito para el periodo de 1999 a 2011, dependiendo de la disponibilidad de información. Se analizaron todos los eventos de tránsito y lesionados por el tránsito de vehículos de motor en Guadalajara y Zapopan. Guadalajara y Zapopan son los municipios más poblados de la Zona Metropolitana de Guadalajara que a su vez constituye la segunda más habitada de México. Tan solo en estos dos municipios vivían 2.835.953 habitantes en 2010 (Instituto Nacional de Estadística y Geografía. Centro de Población y Vivienda, 2010. http://www.inegi.org.mx/est/ contenidos/proyectos/ccpv/cpv2010/Default. aspx, accedido el Abr/2013) y están situados en el occidente de México y al centro del Estado de Jalisco.

\section{Variables y fuentes de información}

En la Tabla 1 se presenta un resumen de todas las fuentes de información utilizadas, el periodo pa- ra el que se tuvo dicha información, así como las limitaciones de cada una de ellas. Se analizaron las tasas mensuales de mortalidad, egresos hospitalarios y ocurrencia de colisiones por 100.000 habitantes; y, además, el porcentaje de muertes por lesiones causadas por el tránsito asociadas con el consumo de alcohol. En la Tabla 2 se muestra la construcción de los indicadores según la fuente de información. Para calcular las tasas, se empleó la población media anual reportada por la Dirección General de Información en Salud (Estimaciones con Base en las Proyeciones de la Población de México, 2005-2030. http://pda.sa lud.gob.mx/cubos, accedido el Abr/2013), como denominador para todos los meses de cada año, ya que no se dispone de una estimación de la población mensual. Los datos de mortalidad se obtuvieron del Instituto Nacional de Estadística Geografía e Información (INEGI) quien integra, valida y reporta los certificados de defunción que cumplimenta el sector salud. Es importante señalar, que a partir de la base de datos de mortalidad, no se puede determinar en cuántos de estos fallecidos había alcohol involucrado. Para ello se empleó información del SISVEA que documenta los resultados toxicológicos de las autopsias realizadas en los Servicios Médicos Forenses (Dirección General de Epidemiología, Subsecretaría de Prevención y Promoción de la Salud, Secretaría de Salud. Información Epidemiológica. Informes Sistema de Vigilancia Epidemiológica de las Adicciones. http://www.epidemiologia.salud.gob. $\mathrm{mx} /$ dgae/infoepid/inf_sisvea.html18, accedido el Abr/2013).

De igual forma, se analizaron las hospitalizaciones por lesiones causadas por el tránsito, reportadas por la Secretaría de Salud para Guadalajara y Zapopan en el periodo de 2007 a 2011. Según cifras oficiales, en Jalisco, el 51\% de los egresos en hospitales públicos se dieron en unidades de la Secretaría de Salud 15. Los egresos hospitalarios de otras instituciones públicas no estuvieron disponibles para el análisis.

Finalmente, se analizó la base de datos de accidentes de tránsito en zonas urbanas y suburbanas (ATUS) (Instituto Nacional de Estadística y Geografía. Accidentes de Tránsito Terrestre en Zonas Urbanas y Suburbanas, 1999-2010. http:// www.inegi.org.mx/sistemas/olap/proyectos/ bd $/$ consulta.asp? $\mathrm{p}=14744 \& \mathrm{c}=23705 \& \mathrm{~s}=\mathrm{est} \& \mathrm{cl}$, accedido el Abr/2013) que integra el INEGI corresponde al periodo 1999 a 2011; y de las llamadas de emergencia, captadas por el Centro de Comando, Control, Comunicaciones y Cómputo (Emergencias 066); se incluyeron en este análisis aquellas llamadas al número de emergencias 066 que se atendieron con motivo de algún evento de tránsito en el periodo de enero de 2005 al 25 de 
Tabla 1

Fuentes de información utilizadas para el análisis del impacto de la reforma en la legislación sobre alcohol y conducción en Guadalajara y Zapopan, Jalisco, México.

\begin{tabular}{|c|c|c|c|}
\hline Fuente & Periodo & Información & Limitantes \\
\hline \multicolumn{4}{|l|}{ Mortalidad } \\
\hline $\begin{array}{l}\text { Instituto Nacional de } \\
\text { Estadística y Geografía } \\
\text { (INEGI) }\end{array}$ & $\begin{array}{l}\text { Enero } 1999 \\
\text { a diciembre } \\
2011\end{array}$ & Base de datos de mortalidad & $\begin{array}{l}\text { No incluye información sobre condición de consumo } \\
\text { de alcohol previo al deceso }\end{array}$ \\
\hline $\begin{array}{l}\text { Sistema de Vigilancia } \\
\text { Epidemiológica de las } \\
\text { Adicciones (SISVEA) }\end{array}$ & $\begin{array}{l}\text { Enero } 1999 \\
\text { a diciembre } \\
2011\end{array}$ & $\begin{array}{l}\text { Resultado toxicológico de las autopsias } \\
\text { realizadas a los fallecimientos causados } \\
\text { por el tránsito }\end{array}$ & $\begin{array}{l}\text { No se determina el municipio donde ocurrió la } \\
\text { colisión, ni en el que sucedió la defunción, tampoco } \\
\text { se cuenta con información detallada sobre la } \\
\text { concentración de alcohol en sangre. Se desconoce si } \\
\text { el lesionado iba conduciendo }\end{array}$ \\
\hline \multicolumn{4}{|l|}{ Hospitalario } \\
\hline Egresos hospitalarios & $\begin{array}{l}\text { Enero } 2007 \\
\text { a diciembre } \\
2011\end{array}$ & $\begin{array}{l}\text { Información sobre egresos hospitalarios } \\
\text { en los hospitales de la Secretaría de } \\
\text { Salud }\end{array}$ & $\begin{array}{l}\text { Sólo incluye información sobre una de las instituciones } \\
\text { públicas, no se incluye información sobre servicios de } \\
\text { salud del IMSS (quienes no registran la causa externa), } \\
\text { hospitales privados, etc. Tampoco se registra la } \\
\text { condición de consumo de alcohol }\end{array}$ \\
\hline \multicolumn{4}{|l|}{ Siniestros viales } \\
\hline $\begin{array}{l}\text { Estadística de accidentes de } \\
\text { tránsito terrestre en zonas } \\
\text { urbanas y suburbanas (ATUS) }\end{array}$ & $\begin{array}{l}\text { Enero } 1999 \\
\text { a diciembre } \\
2011\end{array}$ & $\begin{array}{l}\text { Información sobre siniestros viales por } \\
\text { severidad (sólo choque, choque con } \\
\text { lesionados y choque con fallecidos.) } \\
\text { Se documenta el número de eventos } \\
\text { asociados al alcohol }\end{array}$ & $\begin{array}{l}\text { Importante subregistro de los choques asociados al } \\
\qquad \text { alcohol }\end{array}$ \\
\hline Emergencias 066 & $\begin{array}{c}2005 \text { al } 25 \\
\text { de agosto de } \\
2011\end{array}$ & $\begin{array}{c}\text { Información sobre la respuesta a } \\
\text { incidentes reportados al teléfono de } \\
\text { Emergencias } 066\end{array}$ & $\begin{array}{l}\text { No se menciona la condición de consumo de alcohol } \\
\text { en el momento del evento de tránsito }\end{array}$ \\
\hline
\end{tabular}

IMSS: Instituto Mexicano del Seguro Social.

agosto de 2011 (la base de datos fue solicitada a las autoridades correspondientes, por lo que no existe una página web de referencia). En la medida de lo posible, sólo se consideró una llamada por evento.

La variable independiente comprende las dos intervenciones a evaluar: IMESEVI y los cambios de la legislación en Jalisco. Con este fin, se creó una variable categórica que adquirió valor " 0 " para los meses anteriores a la fecha de inicio de IMESEVI, "1" a partir de marzo de 2008 cuando inició este programa y "2" cuando entraron en vigor las modificaciones a la legislación (septiembre de 2010). En este último periodo, tanto el IMESEVI, como la "Ley Salvavidas", funcionan paralelamente.

\section{Análisis estadístico}

Para el análisis estadístico, se empleó el software Stata 12 (Stata Corp., College Station, Estados Unidos). El efecto del cambio en la legislación se evaluó mediante series de tiempo interrumpidas, cada uno de los indicadores definidos pre- viamente en la Tabla 2 se analizaron por separado. En el análisis de series de tiempo se utilizaron Modelos Autorregresivos Integrados de Medias Móviles (ARIMA, por sus siglas en inglés). Estos, permiten predecir los valores de una variable a partir de sus valores pasados, eliminando el supuesto de independencia de las observaciones bajo estudio 16. Así, cada observación se modela en función de sus valores anteriores 17 .

La definición del mejor modelo para cada indicador siguió la aproximación propuesta por Box-Jenkins, quienes sugirieron emplear un proceso de cuatro pasos 17,18. En una primera instancia, se determinó si la serie de tiempo tenía o no raíz unitaria, es decir, si era o no estacionaria. Con este fin, se empleó la prueba de raíz unitaria de Dickey-Fuller. En un segundo paso, se estimaron los parámetros de los términos autorregresivos y de media móvil del modelo. Posteriormente, se eligió el mejor modelo con ayuda del criterio de Akaike (AIC, por sus siglas en inglés). Para evaluar el ajuste final de los modelos, se empleó la prueba de ruido blanco de Barlett en los residuos estimados. 
Variables de interés para el estudio del impacto de la reforma en la legislación sobre alcohol y conducción en Guadalajara y Zapopan, Jalisco, México.

\begin{tabular}{|c|c|}
\hline Tipo de variable/Indicador & Construcción \\
\hline \multicolumn{2}{|l|}{ Variable independiente } \\
\hline \multirow[t]{4}{*}{ Intervención } & Variable categórica que toma los siguientes valores: \\
\hline & $0=$ periodo previo a febrero 2008 \\
\hline & $1=$ periodo de marzo 2008 a agosto de 2010 \\
\hline & $2=$ de septiembre 2010 a diciembre 2011 \\
\hline \multicolumn{2}{|l|}{ Variables dependientes } \\
\hline \multicolumn{2}{|l|}{ Mortalidad } \\
\hline \multirow{2}{*}{$\begin{array}{l}\text { Tasa de mortalidad por lesiones causadas por el } \\
\text { tránsito }\end{array}$} & Número de muertes por lesiones causadas por el tránsito x 100.000 \\
\hline & Población a mitad del año \\
\hline \multirow[t]{2}{*}{ Muertes asociadas al alcohol (\%) } & Número de autopsias con alcoholemias positivas $\times 100$ \\
\hline & Total de autopsias realizadas a fallecidos por lesiones causadas por el tránsito \\
\hline \multicolumn{2}{|l|}{ Hospitalizaciones } \\
\hline \multirow[t]{2}{*}{$\begin{array}{l}\text { Tasa de hospitalización por lesiones causadas por el } \\
\text { tránsito }\end{array}$} & $\begin{array}{l}\text { Número de egresos hospitalarios por lesiones causadas } \\
\text { por el tránsito en la Secretaría de Salud }\end{array}$ \\
\hline & Población a mitad del año \\
\hline \multicolumn{2}{|l|}{ Siniestros viales } \\
\hline \multirow[t]{2}{*}{ Tasa de colisiones } & Número de colisiones $\times 100.000$ \\
\hline & Población a mitad del año \\
\hline \multirow[t]{2}{*}{ Tasa de llamadas recibidas a Emergencias 066} & Número de eventos independientes reportados $\times 100.000$ \\
\hline & Población a mitad del año \\
\hline
\end{tabular}

Nota: la definición de lesiones causadas por el tránsito, fatales y no fatales, incluyó los siguientes códigos CIE-10, tomando como referencia la definición nacional: V02-V04 (.1-.9), V09.2, V09.3, V09.9, V12-V14 (.3-.9), V19 (.4-.6), V20-V28 (.3-.9), V29- V79 (.4-.9), V80 (.3-.5), V81.1, V82.1, V83-V86 (.0-.3), V87 (.0-.8), V89.2 y V89.9

\section{Consideraciones éticas}

Este trabajo fue revisado y aprobado por los Comités de Ética, Investigación y Bioseguridad del Instituto Nacional de Salud Pública de México. Este estudio se basó en información secundaria, por lo que se consideró un estudio sin riesgo, de acuerdo a los criterios establecidos por el Collaborative Institutional Training Initiative (https:// www.citiprogram.org, accedido el Abr/2013).

\section{Resultados}

En la Tabla 3 se muestra el resultado del análisis de la serie de tiempo de todos los indicadores de interés. Se muestran los procesos autorregresivos (AR) y de medias móviles (MA) que son significativos para el modelo propuesto. En caso de que la serie no fuera estacionaria, se empleó la primera diferencia $[\mathrm{D}(1)]$ para lograr este supuesto del análisis. De acuerdo a lo observado, las series que contaban con una tendencia estacional anual, fueron modeladas bajo estas observaciones [S(12)].
Como se muestra, la tasa de mortalidad mensual por lesiones causadas por el tránsito muestra un aumento no significativo en la tendencia a partir de la implementación de IMESEVI. Con la reforma a la ley, se percibe una reducción en la tendencia, que tampoco es estadísticamente significativa. El análisis del porcentaje de muertes asociadas al alcohol muestra un aumento de 1,9 puntos porcentuales ( $p=0,056$ ), a partir de que se pone en marcha la IMESEVI, y una reducción, estadísticamente significativa, de 5,7 puntos porcentuales $(\mathrm{p}=0,018)$ con la implementación de la “Ley Salvavidas”. Esto se puede observar más claramente en la Figura 1 en la que además se observa que los meses de diciembre 2010 y junio 2011 fueron reportados con $0 \%$ y que prácticamente $60 \%$ de las autopsias realizadas durante mayo 2011 dieron un resultado positivo al alcohol. Este comportamiento es diferente a lo observado en los meses previos.

La tasa de egresos hospitalarios no muestra cambios luego de la implementación de ambas intervenciones (Tabla 3). Sin embargo, se observa una reducción significativa en la tendencia mensual de colisiones con la implementación 
Efecto estimado de la "Ley Salvavidas" en la mortalidad y morbilidad causadas por el tránsito en Guadalajara y Zapopan, Jalisco, México.

\begin{tabular}{|c|c|c|c|}
\hline & Estimador & $\begin{array}{c}\text { Error } \\
\text { estándar }\end{array}$ & Valor de $\mathrm{p}$ \\
\hline \multicolumn{4}{|l|}{ Mortalidad } \\
\hline \multicolumn{4}{|l|}{ Tasa de mortalidad } \\
\hline \multicolumn{4}{|l|}{$\mathrm{AR}(48$ 12) S(12) MA (12) } \\
\hline Implementación de IMESEVI 1era fase & 0,071 & 0,056 & 0,210 \\
\hline Implementación de la nueva ley & $-0,031$ & 0,097 & 0,749 \\
\hline \multicolumn{4}{|c|}{$\begin{array}{l}\text { Porcentaje de muertes producidas en eventos de tránsito con } \\
\text { presencia de alcohol }\end{array}$} \\
\hline \multicolumn{4}{|l|}{$A R(39) S(12) M A(9$ 12) } \\
\hline Implementación de IMESEVI 1era fase & 1,902 & 0,993 & 0,056 \\
\hline Implementación de la nueva ley & $-5,654$ & 2,393 & 0,018 \\
\hline \multicolumn{4}{|l|}{ Hospitalizaciones } \\
\hline \multicolumn{4}{|c|}{ Tasa de hospitalización en hospitales de la Secretaría de Salud } \\
\hline \multicolumn{4}{|l|}{$A R(10) D(1) M A(1)$} \\
\hline Implementación de IMESEVI 1era fase & $-0,181$ & 0,186 & 0,330 \\
\hline Implementación de la nueva ley & $-0,123$ & 0,300 & 0,682 \\
\hline \multicolumn{4}{|l|}{ Eventos viales } \\
\hline \multicolumn{4}{|c|}{ Choques registrados en zonas urbanas y suburbanas } \\
\hline \multicolumn{4}{|l|}{$A R(13$ 12) S(12) MA (1 23410 11) } \\
\hline Implementación de IMESEVI 1era fase & $-9,677$ & 2,980 & 0,001 \\
\hline Implementación de la nueva ley & $-9,932$ & 4,355 & 0,023 \\
\hline \multicolumn{4}{|c|}{ Llamadas recibidas en el teléfono de Emergencias 066} \\
\hline \multicolumn{4}{|l|}{$A R(8$ 12) D(1) MA (1) } \\
\hline Implementación de IMESEVI 1era fase & 0,029 & 1,613 & 0,986 \\
\hline Implementación de la nueva ley & $-0,158$ & 1,321 & 0,905 \\
\hline
\end{tabular}

AR: procesos autorregresivos; IMESEVI: Iniciativa Mexicana de Seguridad Vial; MA: medias móviles.

de IMESEVI ( $\mathrm{p}=0,001)$, y con la "Ley Salvavidas” también se encuentra una reducción significativa $(p=0,023)$. Sin embargo, cabe mencionar que, a pesar de mostrar esta tendencia descendente, en estos dos periodos es superior al periodo basal, previo a IMESEVI. Una de las posibles causas de esta variación en la tendencia podría ser explicada por la calidad de la información. Sin embargo, como se observa en la Figura 2 existe un incremento a partir de agosto 2011 que deberá ser monitoreado con información más reciente.

Finalmente, con la implementación de IMESEVI, se observa un aumento en la tendencia mensual de las llamadas reportando algún evento de tránsito; y con la reforma a la ley, se observa una reducción en la tendencia. Sin embargo, ninguno de estos cambios resultó estadísticamente significativo.

\section{Discusión}

Los resultados de este análisis muestran que a partir de la implementación de la IMESEVI y la "Ley Salvavidas" se ha disminuido significativamente el porcentaje de muertes por lesiones causadas por el tránsito, asociadas con el alcohol, y la tasa mensual de choques en los municipios de Guadalajara y Zapopan. Sin embargo, hasta diciembre de 2011, aún no se observan cambios en términos de las tasas de mortalidad y egresos hospitalarios.

Aunque positivos, estos resultados no son tan alentadores como lo reportado en estudios previos. El impacto de la reducción de los límites permitidos de alcoholemia al conducir, específicamente al nivel de $0,05 \mathrm{~g} / \mathrm{dL}$, ha sido evaluado en Estados Unidos, Francia, Austria, Inglaterra y Gales, mostrando efectos positivos en diversos indicadores, principalmente de mortalidad 6,9. 
Tendencia del porcentaje de muertes por lesiones, causadas por el tránsito en Guadalajara y Zapopan, Jalisco, México, antes y después de la

"Ley Salvavidas", 1999-2011.

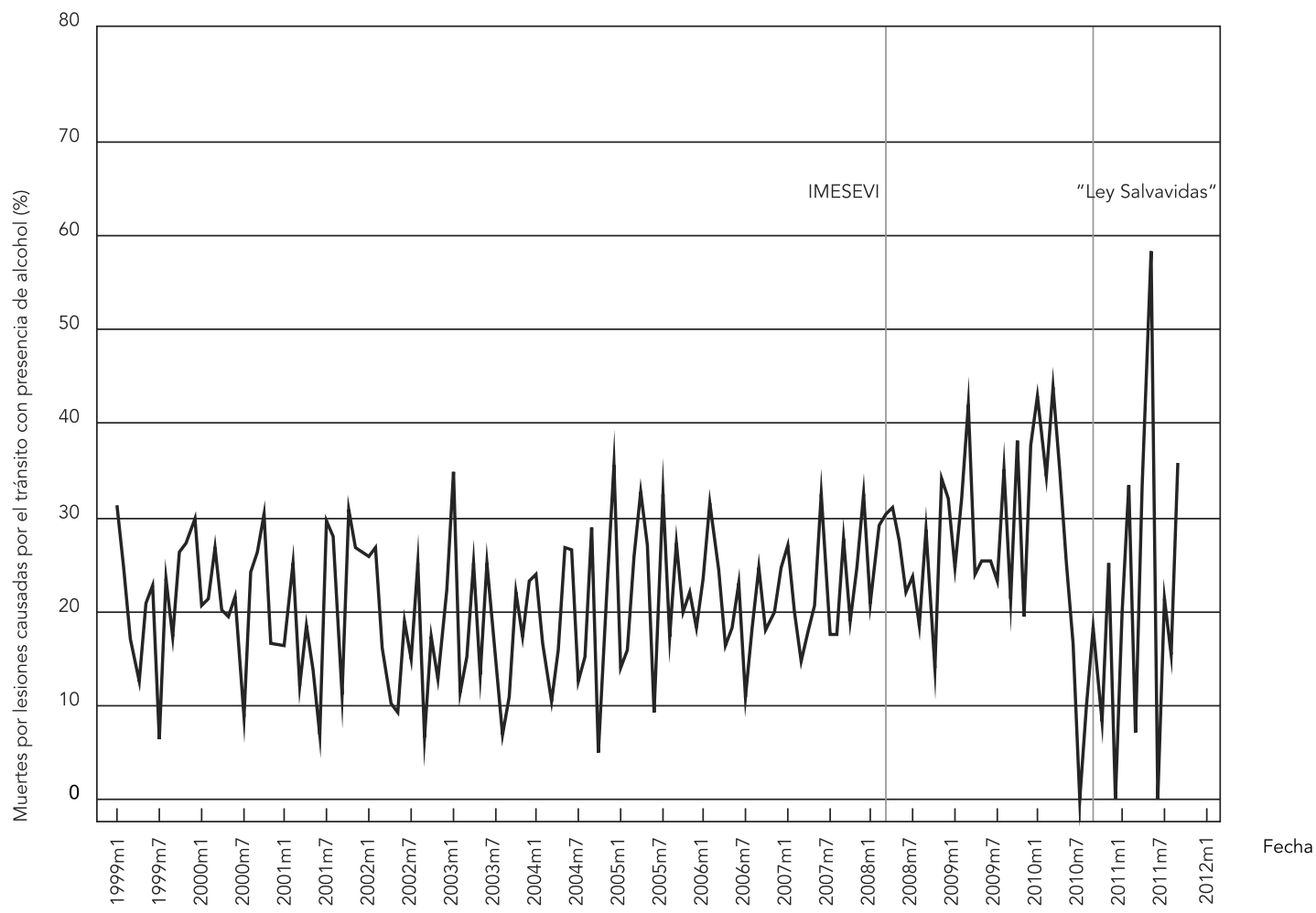

IMESEVI: Iniciativa Mexicana de Seguridad Vial.

Nota: esta figura fue generada con el software Stata 12.

Resultados similares han sido reportados en Australia y los Países Bajos, en donde los efectos han sido atribuidos, en gran parte, a la publicidad y a la aplicación rigurosa de la ley 6,9,19. En Japón, al modificar el límite de alcoholemia a $0,03 \mathrm{~g} / \mathrm{dL}$, se documentaron reducciones significativas en las muertes y lesiones asociadas al beber y conducir, e incluso, la reducción fue observable en todas las lesiones causadas por el tránsito, independientemente de la condición de consumo de alcohol 20. También se han mostrado resultados positivos en Brasil, país de ingresos medios, tras la reducción del nivel de alcoholemia legal a $0,02 \mathrm{~g} / \mathrm{dL}$. Andreuccetti et al. 21 documentaron una reducción significativa en las muertes asociadas con el tránsito un año y medio después. En São Paulo, Brasil, Ribeiro et al. 22 encontraron una disminución del $45 \%$ en la prevalencia de consumo de alcohol y conducción a partir de la aplicación de esta legislación.
Un aspecto clave para lograr incidir en la mortalidad y morbilidad asociadas al tránsito es la aplicación rigurosa de la legislación. Para disuadir a un conductor de beber y conducir, es necesario un sistema legal que asegure una sanción pronta y certera 23 acompañados de acciones de reforzamiento, como las campañas publicitarias 24 y controles aleatorios de alcohol en conductores 25. Mediante tales acciones, es posible influir en la percepción del costo de involucrarse en acciones ilegales, como manejar después de haber bebido 26. Los puntos de alcoholimetría, además de retirar a los conductores ebrios de las calles, aumentan la percepción de riesgo de ser sancionado en la falta, aún en aquellos que no son detenidos 27 . Los controles aleatorios tienen la ventaja de evitar que la detención de los conductores dependa del criterio de los oficiales que apliquen las detenciones 7, pues incluso los más capacitados pueden tener 
Figura 2

Tendencia de la tasa mensual de colisiones de tránsito por 100.000 habitantes en Guadalajara y Zapopan, Jalisco, México, antes y después de la "Ley Salvavidas", 1999-2011.

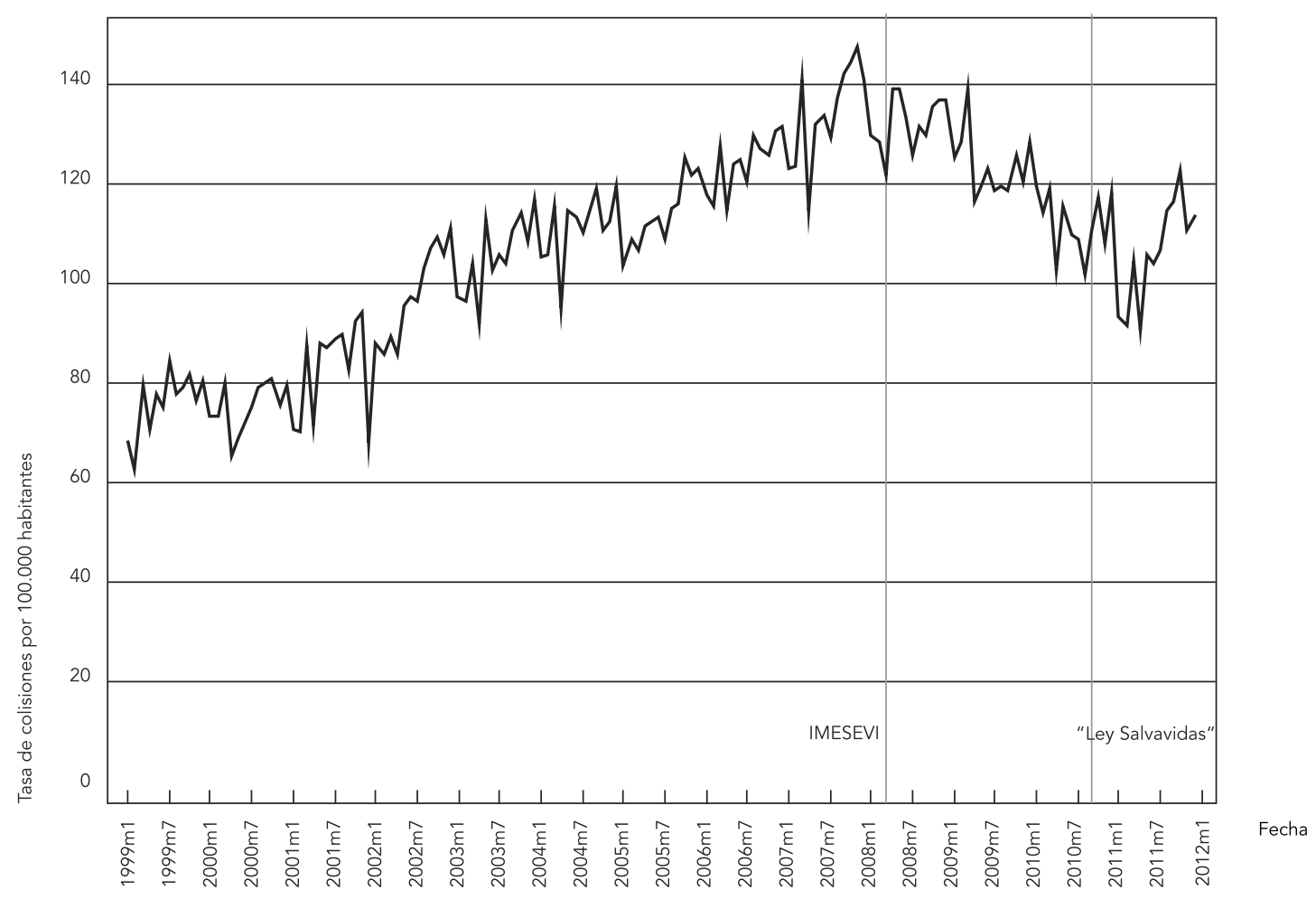

IMESEVI: Iniciativa Mexicana de Seguridad Vial.

Nota: esta figura fue generada con el software Stata 12.

dificultades para identificar los signos de intoxicación de los bebedores "experimentados", que tienen mayor resistencia biológica al alcohol 28,29. La detención aleatoria, realizada de manera frecuente, acompañada de las campañas de comunicación social, puede lograr una mayor disuasión en los conductores, y consecuentemente, lograr un mayor impacto en la reducción de las lesiones asociadas con el consumo de alcohol 25,27. Mientras que las campañas de comunicación social tienen un rol importante, son una estrategia instrumental que si no es acompañada de una correcta aplicación de la legislación, sus efectos positivos desaparecen con el tiempo, como ha sido reportado en otros contextos 30 .

En el contexto particular de Guadalajara y Zapopan podemos identificar problemas en la legislación que limitaban su aplicación, pues hasta antes de agosto 2013 esta no brindaba elementos suficientes a las autoridades locales para realizar controles aleatorios a conductores de vehículos de motor. Durante el periodo bajo estudio, para que al conductor se le realizara la prueba de alcoholimetría, era necesario que incurriera en alguna falta al reglamento de tránsito, lo que pudo haber limitado el impacto de la "Ley Salvavidas”. El 10 de agosto de 2013 se decretó la Ley de Movilidad y Transporte del Estado de Jalisco 31, que si bien sustituye a la "Ley Salvavidas", retoma los límites de alcoholemia modificados anteriormente y llena algunas de las brechas que fueron identificadas durante su implementación. Entre los cambios más importantes están la posibilidad de que la policía de tránsito realice detecciones aleatorias de alcohol en conductores, y agiliza la aplicación de la legislación que ahora incluye el pago de una multa o el arresto administrativo inconmutable que puede ir de 12 a 36 horas, dependiendo del nivel de alcohol detecta- 
do. La reincidencia en un periodo de dos años es penalizada con la suspensión de la licencia para conducir. Consideramos que esto tendrá un impacto positivo en términos de reducir el alto numero de muertos y lesionados en el tránsito en estos municipios.

Para que la ley sea aplicada de manera correcta, los encargados de observarla, en este caso los agentes de tránsito, necesitan de los recursos adecuados, tanto materiales como en capacitación, para detener a los infractores 29 . Se ha observado también que un factor relevante para la realización exitosa de las alcoholimetrías aleatorias es la motivación de las autoridades y sus decisiones para el uso de recursos materiales y humanos. En Estados Unidos se reportó que, en aquellos estados donde se realizaban con mayor frecuencia controles de alcohol aleatorios, la motivación para realizarlos se lograba gracias a una combinación de apoyo de los grupos de trabajo, Organizaciones de la Sociedad Civil, de los oficiales de policía que comprendían la labor de disuasión de los puntos de alcoholimetría, y de la sociedad. También, se identificó que las barreras a su aplicación, se pueden solventar mediante la educación y la capacitación 25. Futuros análisis podrían dar cuenta de cómo la preparación, la capacitación y la motivación de las autoridades están impactando en su trabajo diario y, en el aporte que este tiene a la prevención de choques, lesiones y muertes asociadas al tránsito.

Una ventaja de este estudio fue incluir diversos indicadores para analizar de manera integral la situación de las lesiones causadas por el tránsito en ambos municipios. Se utilizó la mejor información disponible en el momento de la investigación y, con el análisis de series de tiempo, se empleó el mejor abordaje para evaluar estas intervenciones 9,32. Así, nuestros resultados proveen evidencia sobre los efectos de la intervención en el ámbito poblacional, buscando retroalimentar el quehacer de los tomadores de decisiones y los formuladores de políticas públicas. Futuros abordajes podrían valorar la adición de un grupo de control o comparación al análisis de series de tiempo, con el ánimo de enriquecer el análisis y valorar hasta qué punto los hallazgos documentados constituyen una tendencia igualmente observada en otros contextos o no. A pesar de que los modelos de series de tiempo utilizados en este abordaje mostraron un buen ajuste, es importante señalar que existen alternativas estadísticas, como el análisis multivariado de series de tiempo, que no presuponen linealidad y que dada la distribución de algunas de las variables analizadas pudieran ser empleados en el futuro para determinar si dichos resultados son consistentes a lo reportado aquí.
Debido a que esta investigación se basa en el análisis secundario de fuentes de información, el estudio tiene algunas limitaciones. La principal, son los problemas en el registro de la información. Las variaciones observadas en el porcentaje de muertes por tránsito, luego de la implementación de la "Ley Salvavidas", pueden estar influyendo en el resultado que reportamos. Esto pudiera reflejar problemas en el sistema de información en particular, lo que además pudiera estar ocurriendo en las otras fuentes. Es importante dar continuidad a este análisis para observar el comportamiento futuro de estos indicadores y poder tener una mejor perspectiva sobre el impacto de la ley.

Existe información de gran relevancia que no fue incluida en el presente trabajo. La información sobre prevalencia de conducción bajo los efectos del alcohol no fue analizada, dado que esta información no se recoge de forma sistemática. Aunque intentamos analizar el número de colisiones en las que el conductor "responsable" del evento se reporta con aliento alcohólico (información generada por la base de datos de ATUS), a partir de 2008 prácticamente en ningún caso se registra esta información adecuadamente, por lo que finalmente decidimos no incluirla en el presente informe. La información sobre los egresos hospitalarios de otras instituciones públicas y privadas, además de la Secretaría de Salud, no estuvo disponible y, en el caso del Instituto Mexicano del Seguro Social (IMSS) que representa un $35 \%$ del total registrado para el sector público, no se codifica la causa externa de las lesiones. Esto podría considerarse un problema en términos de información, pues no contamos con datos de todas las instituciones que conforman el sector salud y, por tanto, constituye una debilidad para la evaluación de esta intervención.

En términos del análisis, es posible que los cambios en la legislación y su aplicación tengan efectos diferenciados por tipo de actor vial, género 33 y grupo de edad 33,34 , situación que no se analizó en el presente trabajo. Por lo que es necesario emplear análisis desagregados en el futuro para diferentes subgrupos de la población, con el objetivo de identificar un efecto diferenciado por estas variables. Por ejemplo, la intervención podría tener un mayor efecto entre los ocupantes de vehículo de motor, particularmente aquellos que son varones y de grupos de edad jóvenes.

A pesar de estas dificultades, el presente estudio sugiere que la "Ley Salvavidas" pudiera tener efectos positivos en el corto plazo, como la reducción en el porcentaje de muertes por lesiones causadas por el tránsito asociadas con alcohol y en la ocurrencia de eventos viales. Los resultados muestran también que, al menos en el corto pla- 
zo, no se ha logrado impactar sobre la mortalidad y morbilidades asociadas, lo que entreabre la oportunidad para identificar algunas áreas susceptibles de mejora. Entre ellas, consideramos que la aplicación de la legislación será más efectiva ahora que la legislación habilita a la policía de tránsito a implementar detecciones aleatorias en los puntos de alcoholimetría. Idealmente, se deberían intensificar las acciones en los puntos de alcoholimetría, tanto en número como en cobertura, con el fin de disuadir a los conductores de conducir tras haber ingerido alcohol.

\section{Resumo}

Os municípios de Guadalajara e Zapapan, Jalisco, México, têm particidado dos esforços para reduzir a incidência de lesões causadas pelo trânsito ao longo dos anos. Assim, têm participado da Iniciativa Mexicana de Seguridad Vial desde 2008, e em setembro de 2010 entrou em vigor a redução do limite legal de alcoolemia para condução de veículos automotores. Com o objetivo de avaliar o impacto de curto prazo dessas ações na ocorrência de acidentes e lesões relacionados ao consumo de álcool, assim como a gravidade das mesmas, realizou-se análise de séries temporais, com dados secundários das bases de dados oficiais de mortalidade, morbidade e acidentes de trânsito, de modo a analisar a tendência destes. Observou-se alterações significativas no porcentual mensal de mortes associadas ao álcool e na taxa de acidentes a partir da implementação dessas intervenções. São apresentadas diversas recomendações para melhorar a aplicação e os resultados dessa reforma.

Consumo de Bebidas Alcoólicas; Acidentes de Trânsito; Políticas Públicas

\section{Colaboradores}

L. Gómez-García participó del diseño del estudio, análisis estadístico, redacción del primer borrador, aprobación de la versión final. R. Pérez-Núñez y E. Hidalgo-Solórzano colaboraron en el diseño del estudio, análisis e interpretación de los datos, revisión crítica y aprobación del artículo.

\section{Agradecimientos}

Se agradece el apoyo y la asesoría técnica brindada por el MC Aarón Salinas Rodríguez para la preparación de este documento; así como la revisión y los comentarios realizados a versiones previas de este documento por las Dras. Martha Híjar, María de Jesús Valerio Orozco y Anahí Cristina Dreser Mansilla; el Dr. Víctor Hugo Robles Calvillo y el Mtro. José Alberto Muños. Este trabajo es parte de la tesis de maestría de la primera autora con el título "Impacto de la reforma en la legislación sobre consumo de alcohol y conducción en Guadalajara y Zapopan, Jalisco" del programa de Maestría en Ciencias de la Salud-Sistemas de Salud de la Escuela de Salud Pública de México, 2012. Fue parcialmente financiado por el Consejo Nacional de Ciencia y Tecnología a través de una beca (Número de registro 231784) y por el Centro de Investigación en Sistemas de Salud del Instituto Nacional de Salud Pública.

\section{Conflicto de intereses}

Los autores del presente manuscrito declaran que no existe ningún potencial conflicto de interés relacionado con la publicación del artículo. 


\section{Referencias}

1. World Health Organization. Global status report on road safety 2013: supporting a decade of action. Geneva: World Health Organization; 2013.

2. Dirección General de Información en Salud, Secretaría de Salud. Información dinámica: defunciones cifras oficiales definitivas. http://pda.salud. gob.mx/cubos/cprin_mortalidad.html (accedido el Abr/2013).

3. Hijar M, Chandran A, Pérez-Núñez R, Lunnen JC, Rodriguez-Hernandez JM, Hyder AA. Quantifying the underestimated burden of road traffic mortality in Mexico: a comparison of three approaches. Traffic Inj Prev 2012; 13 Suppl 1:5-10.

4. Odero W, Zwi A. Alcohol-related traffic injuries and fatalities in developing countries: a critical review of literature. London: Department of Public Health, London School of Hygiene and Tropical Medicine; 1995.

5. Guanche Garcell H, Martínez Quesada CE, Gutiérrez García F. Efecto del alcohol en la capacidad de conducción de vehículos automotores. Rev Cuba Salud Pública 2007; 33:0-0.

6. Fell JC, Voas RB. The effectiveness of reducing illegal blood alcohol concentration (BAC) limits for driving: Evidence for lowering the limit to .05 BAC. J Safety Res 2006; 37:233-43.

7. Solomon R, Chamberlain E, Abdoullaeva M, Tinholt B. Random breath testing: a Canadian perspective. Traffic Inj Prev 2011; 12:111-9.

8. Peden M, Scurfield R, Sleet D, Mohan D, Hyder AA, Jarawan E, et al. Informe mundial sobre prevención de los traumatismos causados por el tránsito. Washinton DC: Organización Panamericana de la Salud; 2004.

9. Killoran A, Canning U, Doyle N, Sheppard L. Review of effectiveness of laws limiting blood alcohol concentration levels to reduce alcohol-related road injuries and deaths: final report. http://www.nice. org.uk/media/3FE/1A/BloodAlcoholContentEffec tivenessReview.pdf (accedido el Abr/2013).

10. World Health Organization. Global status report on road safety: time for action. Geneva: World Health Organization; 2009.

11. Híjar M, Pérez-Núñez R, Inclán-Valadez C, Silveira-Rodrigues EM. Road safety legislation on the Americas. Rev Panam Salud Pública 2012; 32:70-6.

12. Leenen I, Givaudan M, García G. Iniciativa Mexicana de Seguridad Vial y Prevención de Lesiones en el Tránsito (IMESEVI). Construcción de línea base: reporte final. México DF: Centro Nacional para la Prevención de Accidentes; 2008.

13. Pérez-Núñez R, Híjar M, Celis A, Hidalgo E. Encuesta Nacional de Salud y Nutrición 2012. Evidencia para la política pública en salud. Lesiones causadas por el tránsito: hora de poner freno. Cuernavaca: Secretaría de Salud, Instituto Nacional de Salud Pública; 2013.
14. Consejo Nacional para la Prevención de Acciden tes. Seguridad vial 2010. http://www.cenapra.sa lud.gob.mx/interior/seguridad_vial.html (accedido el Abr/2013).

15. Sistema Nacional de Salud. Boletin de Informacion Estadistica 2009; 29(3).

16. Chatfield C. The analysis of time series: an introduction. Boca Raton: Chapman \& Hall/CRC; 2004.

17. Pérez C. Econometría básica: técnicas y herramientas. Madrid: Pearson Educación; 2007.

18. Gujarati D, Porter DC. Econometría. México DF: McGraw-Hill; 2010.

19. Mathijssen MPM. Drink driving policy and road safety in the Netherlands: a retrospective analysis. Transportation Research Part E: Logistics and Transportation Review 2005; 41:395-408.

20. Nagata T, Setoguchi S, Hemenway D, Perry MJ. Effectiveness of a law to reduce alcohol-impaired driving in Japan. Inj Prev 2008; 14:19-23.

21. Andreuccetti G, Carvalho HB, Cherpitel CJ, Ye Y, Ponce JC, Kahn T, et al. Reducing the legal blood alcohol concentration limit for driving in developing countries: a time for change? Results and implications derived from a time-series analysis (2001-10) conducted in Brazil. Addiction 2011; 106:2124-31.

22. Ribeiro V, Souza R, Duailibi S, Florentino J, Laranjeira R, Pinsky I. The effect of the new traffic law on drinking and driving in São Paulo, Brazil. Accid Anal Prev 2013; 50:622-7.

23. Deshapriya EB, Iwase N. Impact of the 1970 legal BAC $0.05 \mathrm{mg} \%$ limit legislation on drunk-driverinvolved traffic fatalities, accidents and DWI in Japan. Subst Use Misuse 1998; 33:2757-88.

24. Elder RW, Shults RA, Sleet DA, Nichols JL, Thompson RS, Rajab W. Effectiveness of mass media campaigns for reducing drinking and driving and alcohol-involved crashes. Am J Prev Med 2004; 27:57-65.

25. Fell JC, Lacey JH, Voas RB. Soberty checkpoints: evidence of effectiveness is strong, but use is limites. Traffic Inj Prev 2004; 5:220-7.

26. Constant A, Salmi LR, Lafont S, Chiron M, Lagarde E. Road casualties and changes in risky driving behavior in France between 2001 and 2004 among participants in the GAZEL cohort. Am J Public Health 2009; 99:1247-53.

27. Elder RW, Shults RA, Sleet DA, Nichols JL, Zaza S, Thompson RS. Effectiveness of sobriety checkpoints for reducing alcohol-involved crashes. Traffic Inj Prev 2002; 3:266-74.

28. Keall MD, Frith WJ. A method for estimating crash risk associated with driver BAC. Transportation Research Part E: Logistics and Transportation Review 2005; 41:409-20. 
29. Pechansky F, Chandran A. Why don't Northern American solutions to drinking and driving work in southern America? Addiction 2012; 107:1201-6.

30. Silveira A. Use of child restraints in cars in urban areas. Buenos Aires: Luchemos por la Vida; 2011.

31. Gobierno del Estado de Jalisco. Decreto no 24.451/ LX/13, por el que se expide la Ley de Movilidad, Tránsito y Transporte del estado de Jalisco. Guadalajara: Gobierno del Estado de Jalisco; 2013.

32. Tippetts AS, Voas RB, Fell JC, Nichols JL. A metaanalysis of .08 BAC laws in 19 jurisdictions in the United States. Accid Anal Prev 2005; 37:149-61.
33. Kaplan S, Prato CG. Impact of BAC limit reduction on different population segments: a Poisson fixed effect analysis. Accid Anal Prev 2007; 39:1146-54.

34. Desapriya E, Shimizu S, Pike I, Subzwari S, Scime G. Impact of lowering the legal blood alcohol concentration limit to 0.03 on male, female and teenage drivers involved alcohol-related crashes in Japan. Int J Inj Contr Saf Promot 2007; 14:181-7.

Recibido el 27/Jun/2013

Versión final presentada el 02/Dic/2013

Aprobado el 06/Dic/2013 